\title{
The protective effects of magnolol on acute trinitrobenzene sulfonic acid-induced colitis in rats
}

\author{
YONG ZHANG $^{1}$, LI-TANG FU ${ }^{2}$ and FANG TANG ${ }^{1}$ \\ ${ }^{1}$ Department of Traditional Chinese Medicine, Tianjin Medical University General Hospital, Tianjin 300052; \\ ${ }^{2}$ Dingzhou Radi-Glory Bio-Chem Co., Ltd., Baoding, Hebei 073000, P.R. China
}

Received December 21, 2015; Accepted January 6, 2017

DOI: $10.3892 / \mathrm{mmr} .2017 .8321$

\begin{abstract}
The present study aimed to investigate the protective effects of magnolol on acute 2,4,6-trinitrobenzene sulfonic acid (TNBS)-induced colitis, and its underlying mechanisms. Experimental colitis was induced by intracolonic administration of TNBS/ethanol into rats. The model rats were randomly assigned into groups: TNBS, magnolol (high, medium and low doses), and salazosulfapyridine (positive control). All intervention regimens were administered by oral gavage, once a day for 7 consecutive days, $24 \mathrm{~h}$ after colitis induction. Histological and biochemical changes in colonic inflammation were evaluated by hematoxylin and eosin and immunohistochemistry, respectively. Rats treated with all doses of magnolol exhibited decreased colonic myeloperoxidase activity $(\mathrm{P}<0.05$ vs. TNBS), reduced serum levels of proinflammatory cytokines [including interleukin (IL)-6 and IL-17], and downregulated Toll-like receptor-4 (TLR-4) mRNA expression. Histological analysis revealed that medium and high doses of magnolol conferred an anti-inflammatory effect, which was indicated by a decrease in disease activity index, an increase in thymus index, and downregulation of nuclear factor (NF)- $\mathrm{\kappa B}$ p65 mRNA and TLR-4 protein expression. However, only high-dose magnolol significantly ameliorated the elevated colon weight/length ratio. The results of the present study indicate that magnolol exerts protective effects against acute TNBS-induced colitis in rats, and the TLR-4/NF- $\kappa B$ signaling pathway-mediated inhibitory effect on inflammatory cascades may contribute to the protective activity of magnolol.
\end{abstract}

\section{Introduction}

Inflammatory bowel disease (IBD) is an idiopathic inflammatory disorder involving the mucosa and submucosa of

Correspondence to: Dr Fang Tang, Department of Traditional Chinese Medicine, Tianjin Medical University General Hospital, 154 Anshan Road, Tianjin 300052, P.R. China

E-mail: zhongyi3599@sina.com

Key words: 2,4,6-trinitrobenzenesulfonic acid, inflammatory bowel disease, magnolol, cytokines, TLR-4/NF- $\kappa$ B signal pathway the colon. The main clinical manifestations of IBD include abdominal pain, diarrhea and purulent stools. IBD provokes an abnormal, exacerbated immune response in the intestine (1), the initiation and progression of which is likely to be associated with certain internal and external environmental factors (1). As a result, the synthesis and release of various proinflammatory mediators, including reactive oxygen and nitrogen metabolites, eicosanoids, cytokines and chemokines are upregulated in IBD. There is currently no cure for this disorder, and clinical management focuses on downregulation of the exacerbated immune response, using drugs such as aminosalicylates, glucocorticoids and immunosuppressants, such as sulfasalazine; however, long-term use of these drugs often results in serious side effects. Biological drugs, such as infliximab and adalimumab, have shown great potential as therapeutics for IBD; however, their exorbitant costs and potential for serious adverse effects limit their long-term use. Recently, much attention has been focused on the inhibitory effects of some active ingredients from natural herbs on inflammatory responses in human IBD or experimental colitis (2).

Cortex Magnoliae officinalis is an herbal supplement in traditional Chinese medicine, which is commonly used to ameliorate microbial infection, inflammation and gastrointestinal disorders (3). Phytochemical studies have demonstrated that this herb is rich in a large number of bioactive substances, including magnolol and honokiol. Magnolol possesses various biological activities, including antioxidative $(4,5)$, antibacterial (6), anti-inflammatory (7), and antiulcer activities (8). An increasing amount of evidence has suggested that oxidative damage serves a key role in the development of tissue destruction in patients with IBD, and modulating free radical production may represent a novel direction for IBD therapy $(9,10)$. Besides the antioxidative activity, previous studies have also shown an interest in using magnolol for the treatment of acute inflammatory conditions $(11,12)$. The present study aimed to investigate whether magnolol exhibits protective effects in TNBS-induced colitis through its anti-inflammatory activity.

\section{Materials and methods}

Chemicals. Sulfasalazine was purchased from Shanghai Xinyi Jiahua Pharmaceutical Co., Ltd. (Shanghai, China); 
magnolol (purity, 98\%) was purchased from Phytomarker, Ltd. (Tianjin, China); and 2,4,6-trinitrobenzenesulfonic acid (TNBS) was purchased from Sigma-Aldrich (Merck KGaA, Darmstadt, Germany). All other chemicals used were of analytical grade.

Animals and colitis modeling. A total of 48 male Wistar rats (age, 6-8 weeks; weight, 200 $\pm 20 \mathrm{~g}$ ), of specific-pathogen-free grade, were obtained from the Military Medical Science Academy (Tianjin, China). All rats were provided with tap water and food ad libitum, and maintained at a controlled temperature $\left(22 \pm 2^{\circ} \mathrm{C}\right)$ and humidity $(50-70 \%)$. The animals were maintained in a $12 \mathrm{~h}$ light/dark cycle throughout the experiment in the Animal Center of the Radiation Medicine Institute, Chinese Academy of Medical Sciences (Tianjin, China). This study was conducted in compliance with the National Institutes of Health Guidelines for the Care and Use of Laboratory Animals (8th edition, China) and received approval from the Animal Care and Use Committee of Tianjin Medical University (Tianjin, China).

A schematic representation of the experimental process is presented in Fig. 1. Colitis was induced on day 0 according to the method described by Morris et al (13). Briefly, all animals were fasted, with ad libitum access to tap water, for $24 \mathrm{~h}$ prior to the induction of colitis. Rats were kept in light narcosis by the administration of $10 \%$ chloral hydrate [300 mg/kg, intraperitoneal (i.p.)]. A modified urinary catheter with an external diameter of $2 \mathrm{~mm}$ was inserted through the rectum and into the colon $\sim 8 \mathrm{~cm}$ proximal to the anus. TNBS dissolved in 50\% ethanol was introduced into the colon through the catheter at a dose of $100 \mathrm{mg} / \mathrm{kg}$ in the colitis groups. Saline with vehicle (ethanol) was given to normal control rats. Animal behavior, body weight, stool consistency and fecal occult blood tests were monitored daily throughout the experiment.

Animals were assigned to six groups using a random number table method ( $\mathrm{n}=8 /$ group). A total of $24 \mathrm{~h}$ after the induction of colitis, doses equivalent to 15,30 and $60 \mathrm{mg} / \mathrm{kg}$ body weight (bw) magnolol were suspended in 5\% sodium carboxymethyl cellulose (CMC-Na) solution. Low-, medium- and high-doses magnolol groups received doses of 15,30 and $60 \mathrm{mg} / \mathrm{kg}$ bw magnolol, respectively. Rats in the normal control and TNBS groups received a comparable volume of the 5\% CMC-Na vehicle solution. Positive control rats received sulfasalazine $(500 \mathrm{mg} / \mathrm{kg}$ bw). Drugs were administered once daily by oral gavage $(10 \mu \mathrm{l} / \mathrm{g}$ bw). All intervention regimens lasted for 7 days. On the 8th day, after a $24 \mathrm{~h}$ fast, the abdominal cavity was opened via a middle incision under chloral hydrate $(300 \mathrm{mg} / \mathrm{kg} \mathrm{bw})$ anesthesia, and blood was collected using the abdominal aortic method, as previously described (13), from which the serum was separated after centrifugation at $1,500 \mathrm{x} \mathrm{g}$ at $4^{\circ} \mathrm{C}$ and stored at $-80^{\circ} \mathrm{C}$ until further analysis. All animals were anaesthetized by $10 \%$ chloral hydrate and were sacrificed by cervical dislocation, and the intestine between the ileocecal junction and anus was excised. The weight/length $(\mathrm{mg} / \mathrm{cm})$ ratio was calculated and the colon was immediately cut into sections. One segment $(1 \mathrm{~cm})$ was fixed in $4 \%$ buffered formaldehyde for histopathological examination; an additional $1 \mathrm{~cm}$ longitudinal piece was immediately snap-frozen in liquid nitrogen and stored at $-80^{\circ} \mathrm{C}$

\begin{tabular}{|c|c|c|c|c|c|c|c|c|}
\hline Day 0 & 1 & 2 & 3 & 4 & 5 & 6 & 7 & 8 \\
\hline$\Delta \dot{z}$ & $\Delta \bullet$ & $\Delta \cdot$ & $\Delta \cdot$ & $\Delta \bullet$ & $\Delta \cdot$ & $\Delta \bullet$ & $\Delta \bullet$ & $\Delta \cdot$ \\
\hline \multicolumn{6}{|c|}{$\begin{array}{l}\Delta: \text { Induction of colitis } \cdot \text { : Oral gavage } \\
\Delta: \text { In fasting stage } \quad \Delta: \text { In feeding stage }\end{array}$} & & & \\
\hline
\end{tabular}

Figure 1. Schematic diagram of the experimental procedure.

for further biochemical studies. The spleen and thymus were also excised and weighed.

Evaluation of disease activity index (DAI). The DAI score was determined by combining scores of: i) Body weight loss, ii) stool consistency, and iii) fecal occult blood as previously described (13). The average of the three values was defined as the DAI. The body weight recorded on day 0 was considered as the baseline, and body weight loss was calculated as the percent difference between the initial body weight at baseline and the body weight on the measurement day. Fecal occult blood testing of stool samples was performed to detect occult bleeding using a commercial kit (BA-2020B; Baso Diagnostics, Inc., Zhuhai, China) following the manufacturer's instructions.

Evaluation of visceral indexes. The visceral index (spleen index/thymus index) was defined as the ratio of the spleen/thymus weight $(\mathrm{mg})$ to body weight $(\mathrm{g})$, whereas the colon weight/length ratio was calculated as the ratio of colonic sample weight $(\mathrm{mg})$ to length $(\mathrm{cm})$.

Macroscopic scoring and histopathological study. The colon mucosal damage index (CMDI) was scored on a 0-8 scale by two observers blinded to treatments, according to the criteria previously described (14), to evaluate any macroscopic colonic damage caused by inflammation. Cross-sections were selected and embedded in paraffin, and $5 \mu \mathrm{m}$ serial sections were obtained and stained with hematoxylin and eosin. The tissue damage index (TDI) was assessed in accordance with previously described criteria (14). Five different regions were selected at random and examined under a Leica DM3000 light microscope (Leica Microsystems GmbH, Wetzlar, Germany) with x100 magnification, and images were captured using a Leica DFC-420C Digital Camera system (Leica Microsystems $\mathrm{GmbH}$ ).

Measurement of myeloperoxidase (MPO) activity. The protein was extracted by RIPA cell lysate (containing PMSF; Thermo Fisher Scientific, Inc., Waltham, MA, USA). Protein concentration was detected using a modified bicinchoninic acid method with a commercially available protein assay kit (Sangon Biotech Co., Ltd., Shanghai, China), according to the manufacturer's instructions. An MPO detection kit (A044; Nanjing Jiancheng Biochemical Engineering Research Institute, Nanjing, China) was used to determine MPO activity following the instruction of the kit, using an Evolution 201 UV-Visible spectrophotometer (Thermo Fisher Scientific, Inc.).

Enzyme-linked immunosorbent assay (ELISA). Serum levels of interleukin (IL)-6 and IL-17 were quantified using 
Table I. Primer sequences for reverse transcription-quantitative polymerase chain reaction analysis.

\begin{tabular}{|c|c|c|}
\hline Gene & Primer sequence (5'-3') & Product size (bp) \\
\hline iNOS & $\begin{array}{l}\text { F: ACATCAGGTCGGCCATCACT } \\
\text { R: CGTACCGGATGAGCTGTGAATT }\end{array}$ & 87 \\
\hline COX-2 & $\begin{array}{l}\text { F: TGTATGCTACCATCTGGCTTCGG } \\
\text { R: GTTTGGAACAGTCGCTCGTCATC }\end{array}$ & 94 \\
\hline TLR-4 & $\begin{array}{l}\text { F: AATCCCTGCATAGAGGTACTTCCTAAT } \\
\text { R: CTCAGATCTAGGTTCTTGGTTGAATAAG }\end{array}$ & 107 \\
\hline $\mathrm{NF}-\kappa \mathrm{B}$ p65 & $\begin{array}{l}\text { F: ACCTGGAGCAAGCCATTAGC } \\
\text { R: CGGACCGCATTCAAGTCATA }\end{array}$ & 100 \\
\hline$\beta$-actin & $\begin{array}{l}\text { F: GTCAGGTCATCACTATCGGCAAT } \\
\text { R: AGAGGTCTTTACGGATGTCAACGT }\end{array}$ & 147 \\
\hline
\end{tabular}

F, forward; R, reverse; iNOS, inducible nitric oxide synthase; COX-2, cyclooxygenase-2; TLR-4, Toll-like receptor-4; NF- $\mathrm{B}$ p65, nuclear factor- $\mathrm{kB}$ p65.

ELISA (IL-16, D1600; IL-17, DY177; R\&D Systems, Inc., Minneapolis, MN, USA) according to the manufacturer's instructions.

Reverse transcription-quantitative polymerase chain reaction (RT-qPCR). Total RNA was extracted from 50-100 mg colonic tissue using TRIzol reagent (Invitrogen; Thermo Fisher Scientific, Inc.). The concentrations and purity of samples were determined using a NanoDrop ${ }^{\mathrm{TM}} 2000$ spectrophotometer (NanoDrop; Thermo Fisher Scientific, Inc., Wilmington, DE, USA), and $2 \mu \mathrm{g}$ RNA was reverse transcribed using a HiFi-MMLV cDNA kit (CW0744; Beijing ComWin Biotech Co., Ltd., Beijing, China) following the manufacturer's instructions. qPCR amplification and detection was performed using a 7500 fast Real-Time PCR system (Applied Biosystems; Thermo Fisher Scientific, Inc.). The SYBR $^{\circledR}$ Premix Ex Taq ${ }^{\mathrm{TM}}$ II (Takara Bio, Inc., Otsu, Japan) and specific primers were annealed at $60^{\circ} \mathrm{C}$. A protocol of $94^{\circ} \mathrm{C}$ denaturation $45 \mathrm{sec}, 59^{\circ} \mathrm{C}$ annealing $45 \mathrm{sec}, 72^{\circ} \mathrm{C}$ extension $60 \mathrm{sec}$ for 32 cycles followed. The specific primers used for inducible nitric oxide synthase (iNOS), cyclooxygenase-2 (COX-2), Toll-like receptor-4 (TLR-4), nuclear factor- $\kappa \mathrm{B}$ p65 $(\mathrm{NF}-\kappa \mathrm{B} \mathrm{p} 65)$ and the $\beta$-actin endogenous control are presented in Table I. Melting curve analysis was performed on each sample to ensure single amplification, and quantification cycle $(\mathrm{Cq})$ values were measured. The $\Delta \mathrm{Cq}$ value was calculated for each sample by subtracting the $\mathrm{Cq}$ value of the endogenous control from the $\mathrm{Cq}$ value of the targeted gene (iNOS, COX-2, TLR-4 and NF- $\mathrm{B}$ p 65 ). The relative expression levels of targeted genes were calculated using the $2^{-\Delta \Delta \mathrm{Cq}}$ method (15).

Immunohistochemistry. Immunohistochemical analysis was performed using the streptavidin-biotin-peroxidase method as previously described (16). Briefly, colonic segments were fixed in $4 \%$ buffered formaldehyde, dehydrated in graded ethanol, embedded in paraffin, and cut into $5 \mu \mathrm{m}$ sections. All sections were incubated with a buffered blocking solution containing normal goat serum (Beyotime Institute of Biotechnology, Haimen, China) for $15 \mathrm{~min}$, which was followed by co-incubation with the primary antibody (TLR-4, cat. no. BA1717, rabbit polyclonal; Wuhan Boster Biological Technology, Ltd., Wuhan, China) diluted 1:100, in a humidity chamber at $37^{\circ} \mathrm{C}$ for $3 \mathrm{~h}$. After washing the sections with phosphate-buffered saline, the sections were incubated with secondary antibody (cat. no. BA1003, biotinylated goat anti-rabbit; Beijing Zhongshan Golden Bridge Biotechnology Co., Ltd., Beijing, China) at $37^{\circ} \mathrm{C}$ for $15 \mathrm{~min}$. Subsequently, sections were rinsed with PBS and co-incubated with horseradish peroxidase-conjugated streptavidin (cat. no. BA1088; Wuhan Boster Biological Technology, Ltd., Wuhan, China) at $37^{\circ} \mathrm{C}$ for $15 \mathrm{~min}$. Sections were counterstained with Harris hematoxylin for $2 \mathrm{~min}$ as previously described (13) and then images were captured under a Leica DM3000 microscope (Leica Microsystems $\mathrm{GmbH}$ ). Five random microscopic fields per section were captured at $\mathrm{x} 400$ magnification, and a threshold optical density was obtained using the Image-Pro Plus 5.1 software (Media Cybernetics, Inc., Rockville, MD, USA). For all analyses, total pixel intensity was determined, and data were presented as optical densities.

Statistical analysis. The results were expressed as the mean \pm standard error. Statistical analysis was performed using SPSS 13.0 (SPSS, Inc., Chicago, IL, USA). One-way analysis of variance and least significant difference multiple comparison tests were used for data analysis. $\mathrm{P}<0.05$ was considered to indicate a statistically significant difference.

\section{Results}

Magnolol protects against TNBS-induced colitis. A total of $24 \mathrm{~h}$ after intracolonic instillation of TNBS/ethanol, rats exhibited piloerection, hypomotility and debilitation. Furthermore, TNBS/ethanol-treated rats suffered from severe anorexia, which was followed by a sharp decline in food intake and a gradual loss of body weight from day 1 to 3 (Fig. 2). These rats developed severe diarrhea on day 2 after TNBS 
Table II. Effects of magnolol on visceral indexes and colonic weight/length ratios in rats, 7 days after the induction of colitis with TNBS.

\begin{tabular}{|c|c|c|c|}
\hline Treatment group & Spleen index & Thymus index & Colonic weight/length ratio $(\mathrm{mg} / \mathrm{cm})$ \\
\hline $\mathrm{N}$ & $0.231 \pm 0.015$ & $0.126 \pm 0.016$ & $8.03 \pm 0.57$ \\
\hline $\mathrm{C}$ & $0.234 \pm 0.051$ & $0.063 \pm 0.021^{\mathrm{a}}$ & $15.43 \pm 2.54^{\mathrm{a}}$ \\
\hline $\mathrm{S}$ & $0.254 \pm 0.027$ & $0.103 \pm 0.027^{\mathrm{b}}$ & $13.14 \pm 2.89^{\mathrm{a}}$ \\
\hline $\mathrm{L}$ & $0.228 \pm 0.034$ & $0.083 \pm 0.025^{\mathrm{a}}$ & $14.10 \pm 1.36^{\mathrm{a}}$ \\
\hline M & $0.249 \pm 0.033$ & $0.102 \pm 0.020^{\mathrm{b}}$ & $13.09 \pm 2.19^{\mathrm{a}}$ \\
\hline $\mathrm{H}$ & $0.251 \pm 0.036$ & $0.117 \pm 0.037^{\mathrm{b}}$ & $11.19 \pm 1.30^{\mathrm{a}, \mathrm{c}}$ \\
\hline
\end{tabular}

Data are presented as the mean \pm standard error. ${ }^{\mathrm{a}} \mathrm{P}<0.01$ vs. normal control group; ${ }^{\mathrm{b}} \mathrm{P}<0.01$ and ${ }^{\mathrm{c}} \mathrm{P}<0.05$ vs. model control group. $\mathrm{N}$, normal control; C, model control; S, sulfasalazine group; L, low (15 mg/kg) magnolol dose; M, medium (30 mg/kg) magnolol dose; H, high (60 mg/kg) magnolol dose.

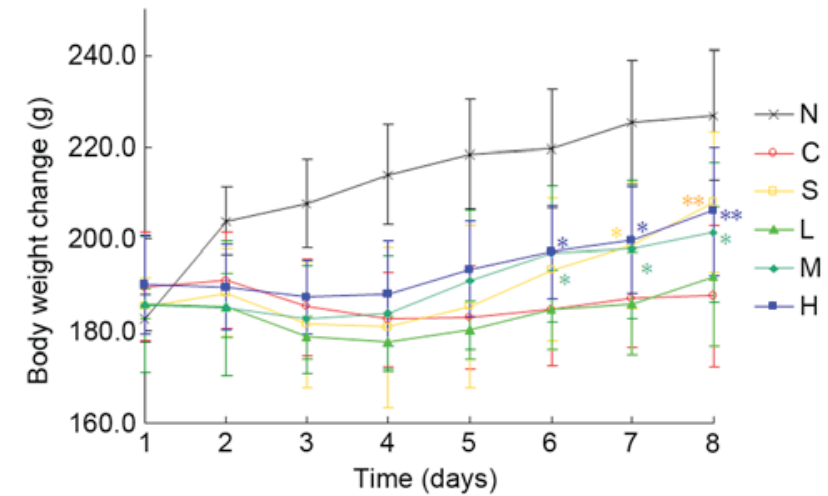

Figure 2. Body weight in different treatment groups. ${ }^{*} \mathrm{P}<0.05$ and ${ }^{* *} \mathrm{P}<0.01$ vs. model control group. $\mathrm{N}$, normal control; $\mathrm{C}$, model control; $\mathrm{S}$, sulfasalazine group; L, low (15 mg/kg) magnolol dose; $\mathrm{M}$, medium (30 mg/kg) magnolol dose; $\mathrm{H}$, high $(60 \mathrm{mg} / \mathrm{kg})$ magnolol dose.

challenge, and rectal bleeding was occasionally observed. A marked increase in DAI score from day 1 was observed in TNBS/ethanol-treated rats compared with the normal control group $(\mathrm{P}<0.01$; Fig. 3$)$. When compared with the TNBS control group, administration of medium- or high-dose magnolol, or $500 \mathrm{mg} / \mathrm{kg}$ sulfasalazine, resulted in a significant reduction in DAI scores $(\mathrm{P}<0.01$ or $\mathrm{P}<0.05)$.

Intracolonic administration of TNBS/ethanol markedly increased the colonic weight/length ratio $(\mathrm{P}<0.01$; Table II). All drug treatments exhibited inhibitory effects on the TNBS-induced increase in colonic weight/length ratio, however only high-dose magnolol significantly reduced the elevated colonic weight/length ratio compared with the TNBS control group $(\mathrm{P}<0.05)$. None of the drugs counteracted the increase in spleen index $(\mathrm{P}>0.05)$, whereas the thymus index was reduced by $\sim 50 \%$ in the TNBS control rats compared with normal control rats. Notably, all interventions, with the exception of low-dose magnolol, resulted in a marked amelioration of the thymus index $(\mathrm{P}<0.01)$.

Macroscopic observation demonstrated colonic mucosal damage with edema, hyperemia, deep ulcerations and focal adhesions to adjacent organs in the TNBS control group (Fig. 4). Conversely, no macroscopic damage was detected in the normal control group. Intracolonic instillation of

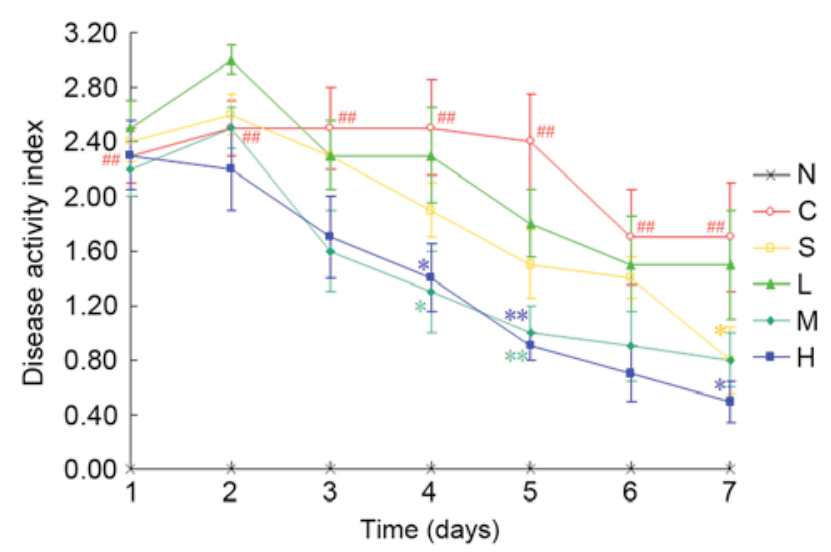

Figure 3. Magnolol ameliorates 2,4,6-trinitrobenzenesulfonic acid/ethanol-induced upregulation of disease activity index. ${ }^{*} \mathrm{P}<0.05$ and ${ }^{* *} \mathrm{P}<0.01$ vs. model control group; ${ }^{\# \#} \mathrm{P}<0.01$ vs. normal control group. $\mathrm{N}$, normal control; C, model control; S, sulfasalazine group; L, low $(15 \mathrm{mg} / \mathrm{kg})$ magnolol dose; $\mathrm{M}$, medium (30 mg/kg) magnolol dose; $\mathrm{H}$, high $(60 \mathrm{mg} / \mathrm{kg})$ magnolol dose.

TNBS/ethanol markedly enhanced CMDI scores $(\mathrm{P}<0.01$ vs. normal control group) (Fig. 4G). CMDI was improved substantially by magnolol when administered at doses of 30 and $60 \mathrm{mg} / \mathrm{kg}(\mathrm{P}<0.05)$. However, $15 \mathrm{mg} / \mathrm{kg}$ magnolol and $500 \mathrm{mg} / \mathrm{kg}$ sulfasalazine failed to mitigate macroscopic damage compared with the TNBS control group ( $\mathrm{P}>0.05)$.

Compared with the normal group (Fig. 5A), the colonic samples from the TNBS control group (Fig. 5B) revealed extensive ulceration and inflammation involving all of the intestinal layers. This inflammatory process was also associated with severe goblet cell depletion. Different degrees of remission were observed in all intervention groups with respect to the TNBS control group. The $60 \mathrm{mg} / \mathrm{kg}$ magnolol group demonstrated the lowest TDI compared with the TNBS control group (Fig. 5G); however, no difference was observed in TDI between the $15 \mathrm{mg} / \mathrm{kg}$ magnolol group and the TNBS control group $(\mathrm{P}>0.05)$.

Effects of magnolol on colonic MPO activity in TNBS-induced colitis. MPO activity was significantly elevated upon stimulation with TNBS/ethanol in colonic tissues $(\mathrm{P}<0.05$; Table III). 

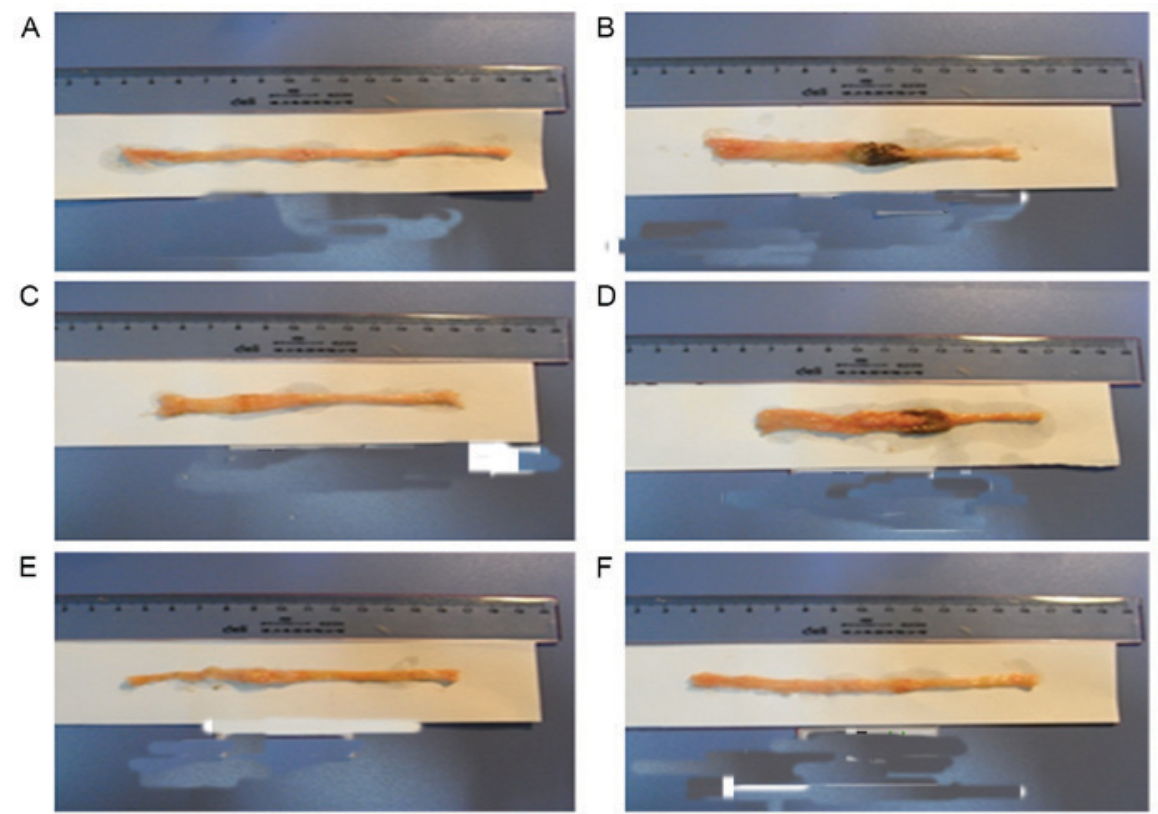

G

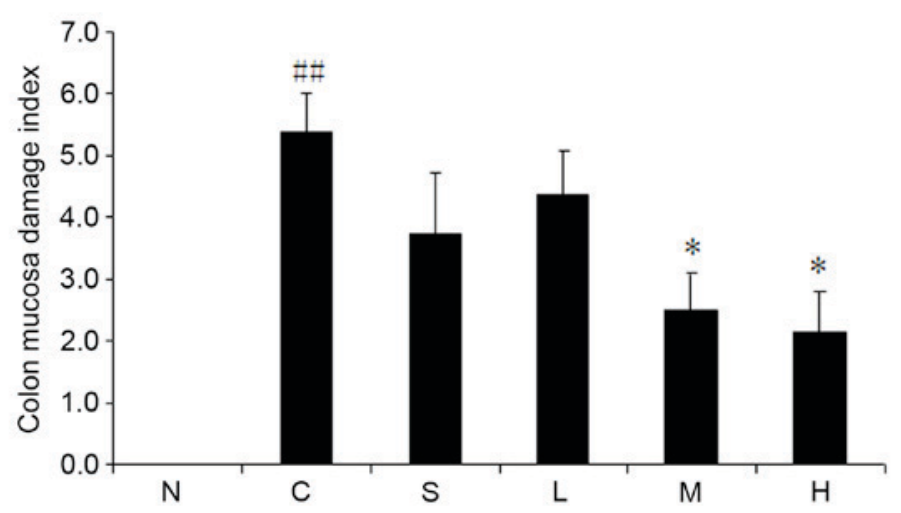

Figure 4. Magnolol ameliorates 2,4,6-trinitrobenzenesulfonic acid/ethanol-induced upregulation of colonic weight/length ratio. (A) Normal control, (B) model control and (C) sulfasalazine groups, and (D) low dose (15 mg/kg), (E) medium dose (30 mg/kg) and (F) high dose (60 mg/kg) magnolol-treated groups. (G) Pooled data from each treatment group. " $\mathrm{P}<0.05$ vs. model control group; ${ }^{\# \#} \mathrm{P}<0.01 \mathrm{vs}$. normal control group. N, normal control; $\mathrm{C}$, model control; $\mathrm{S}$, sulfasalazine group; L, low (15 mg/kg) magnolol dose; M, medium (30 mg/kg) magnolol dose; $\mathrm{H}$, high (60 mg/kg) magnolol dose.

Treatment with 15,30 or $60 \mathrm{mg} / \mathrm{kg}$ magnolol, or $500 \mathrm{mg} / \mathrm{kg}$ sulfasalazine, resulted in decreased MPO activity $(\mathrm{P}<0.05$ vs. TNBS control group).

Effects of magnolol on serum IL-6 and IL-17 levels in rats with TNBS-induced colitis. Colonic injury by TNBS administration was characterized by an increase in the production of the proinflammatory cytokines IL- 6 and IL-17. The levels of these cytokines were significantly reduced in rats treated with all doses of magnolol, or $500 \mathrm{mg} / \mathrm{kg}$ sulfasalazine (Table III).

Effects of magnolol on iNOS, COX-2, TLR-4 and NF- $\kappa B$ p65 mRNA expression levels. The expression of inflammation-related genes was detected following magnolol treatment. iNOS, COX-2, TLR-4 and NF- $\mathrm{B}$ p 65 were all detected at the mRNA level (Fig. 6). TLR-4 gene expression in the TNBS control group was considerably upregulated compared with the normal control group (Fig. 6C), and all tested drugs significantly decreased the expression of TLR- 4 after TNBS instillation $(\mathrm{P}<0.05)$. Similarly, intracolonic instillation of TNBS enhanced the gene expression of iNOS and COX-2 compared with the normal control group (Fig. 6A and B). All tested drugs statistically inhibited the expression of these two genes $(\mathrm{P}<0.05)$, with the exception of $15 \mathrm{mg} / \mathrm{kg}$ magnolol $(\mathrm{P}>0.05)$. TNBS stimulation also elevated NF- $\mathrm{kB}$ p 65 mRNA expression, however only 30 or $60 \mathrm{mg} / \mathrm{kg}$ magnolol, or $500 \mathrm{mg} / \mathrm{kg}$ sulfasalazine, downregulated the increase in NF- $\kappa B$ p65 gene expression $(\mathrm{P}<0.05)$.

Effects of magnolol on TLR-4 protein expression in colonic tissue. TLR-4 expression was further investigated using immunohistochemistry. Protein expression of TLR-4 was predominantly localized within the cell membrane of the colonic mucosal epithelial cells (Fig. 7). In parallel with its gene expression profile, expression of the TLR-4 protein in the TNBS control group was significantly upregulated compared with the normal control group $(\mathrm{P}<0.01)$. However, 30 or $60 \mathrm{mg} / \mathrm{kg}$ magnolol, or $500 \mathrm{mg} / \mathrm{kg}$ sulfasalazine, reduced the protein expression of TLR-4 $(\mathrm{P}<0.05)$. 
A

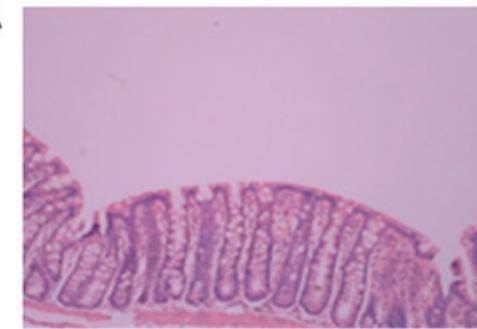

C

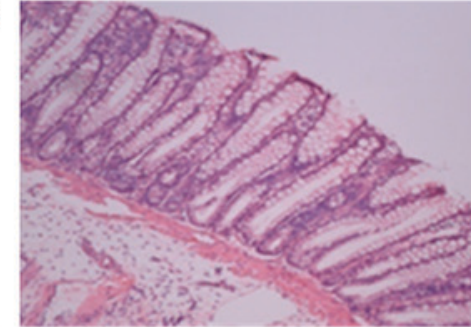

E

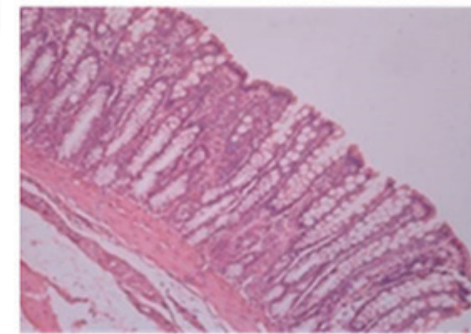

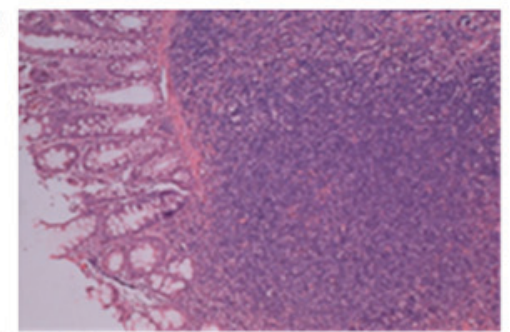

D

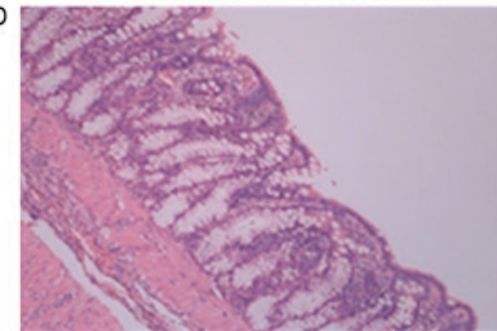

$\mathrm{F}$

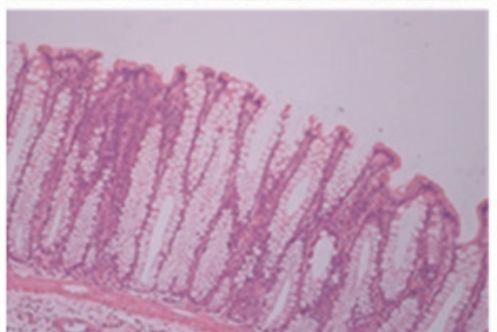

G

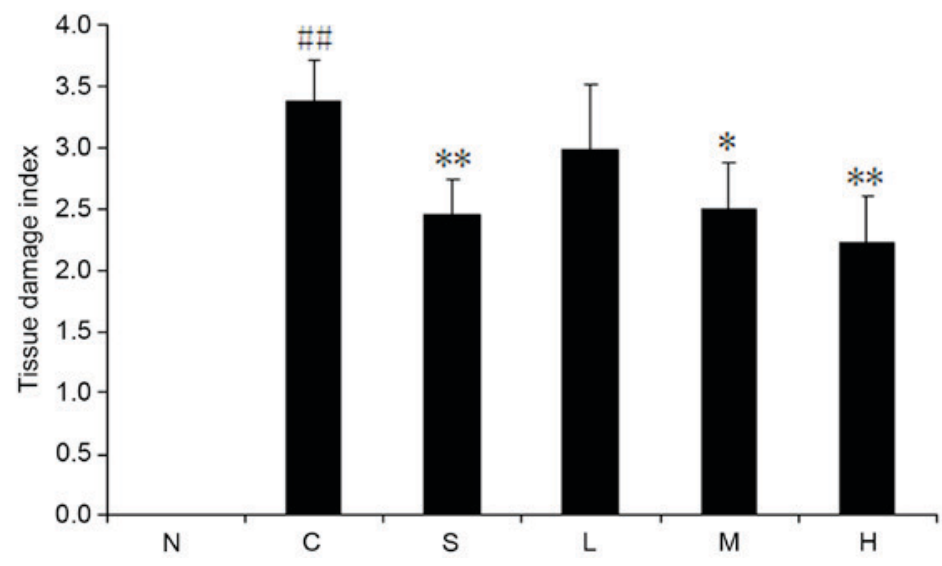

Figure 5. Magnolol ameliorates 2,4,6-trinitrobenzenesulfonic acid/ethanol-induced ulceration and inflammation. (A) Normal control, (B) model control and (C) sulfasalazine groups, and (D) low dose (15 mg/kg), (E) medium dose $(30 \mathrm{mg} / \mathrm{kg})$ and (F) high dose $(60 \mathrm{mg} / \mathrm{kg})$ magnolol-treated groups. (G) Pooled data from each treatment group. ${ }^{*} \mathrm{P}<0.05,{ }^{* *} \mathrm{P}<0.01$ vs. model control group; ${ }^{\sharp *} \mathrm{P}<0.01$ vs. normal control group. $\mathrm{N}$, normal control; $\mathrm{C}$, model control; $\mathrm{S}$, sulfasalazine group; L, low (15 mg/kg) magnolol dose; M, medium (30 mg/kg) magnolol dose; $\mathrm{H}$, high (60 mg/kg) magnolol dose.

\section{Discussion}

The present study demonstrated that magnolol exerts beneficial effects in a dose-dependent manner on TNBS-induced colitis. Anti-inflammatory effects were exerted by magnolol at 30 and $60 \mathrm{mg} / \mathrm{kg}$ doses, and these effects were comparable to those obtained with $500 \mathrm{mg} / \mathrm{kg}$ sulfasalazine, one of the drugs commonly employed in the treatment of human IBD.

Prior to the measurement of biochemical parameters, the clinical effects of magnolol were evaluated by assessing DAI, viscera indexes, macroscopic colonic scores (CMDI) and colonic histopathological scores (TDI). Treating the rats with magnolol for 7 consecutive days resulted in significant amelioration, to varying degrees, in DAI, thymus index, colonic weight/length ratio, CMDI and TDI values. TNBS-induced colonic inflammation is frequently associated with a significant increase in the colonic weight/length ratio, which serves as a reliable indicator of tissue edema (17). In the present study, an inhibitory effect on colonic weight/length ratio was only obtained in the $60 \mathrm{mg} / \mathrm{kg}$ magnolol group; no significant reduction in this ratio was observed in the $500 \mathrm{mg} / \mathrm{kg}$ sulfasalazine group. Therefore, it may be hypothesized that a high dose of magnolol may exert a prominent inhibitory effect on edema and/or fibrosis in the colonic submucosa, which would limit the increase in the colonic weight/length ratio.

The spleen and the thymus, two main immune organs, serve vital roles in inflammatory and immune reactions. Spleen and thymus indexes were therefore calculated to evaluate the immunomodulatory effect of magnolol on TNBS-stimulated rats. Previous research reported that the 
Table III. Effects of magnolol on colonic MPO and serum cytokine activity in rats with 2,4,6-trinitrobenzenesulfonic acid-induced colitis.

\begin{tabular}{lccc}
\hline Treatment group & MPO activity (U/g protein) & IL-6 (ng/l) & IL-17 (pg/l) \\
\hline N & $14.99 \pm 1.39$ & $41.44 \pm 6.06$ & $26.05 \pm 1.62$ \\
C & $21.07 \pm 3.74^{\mathrm{a}}$ & $64.37 \pm 4.21^{\mathrm{a}}$ & $33.07 \pm 1.29^{\mathrm{a}}$ \\
$\mathrm{S}$ & $15.95 \pm 2.41^{\mathrm{a}}$ & $48.82 \pm 10.95^{\mathrm{b}}$ & $26.78 \pm 2.62^{\mathrm{b}}$ \\
$\mathrm{L}$ & $18.43 \pm 2.55^{\mathrm{b}}$ & $49.52 \pm 9.73^{\mathrm{b}}$ & $28.37 \pm 3.22^{\mathrm{b}}$ \\
$\mathrm{M}$ & $15.38 \pm 1.76^{\mathrm{b}}$ & $46.71 \pm 10.07^{\mathrm{b}}$ & $27.28 \pm 2.30^{\mathrm{c}}$ \\
$\mathrm{H}$ & $14.74 \pm 2.15^{\mathrm{b}}$ & $45.41 \pm 3.61^{\mathrm{c}}$ & $27.07 \pm 2.06^{\mathrm{c}}$ \\
\hline
\end{tabular}

Data are presented as the mean \pm standard error. ${ }^{\mathrm{a}} \mathrm{P}<0.01 \mathrm{vs}$. normal control group; ${ }^{\mathrm{b}} \mathrm{P}<0.05$ and ${ }^{\mathrm{c}} \mathrm{P}<0.01$ vs. model control group. MPO, myeloperoxidase; IL, interleukin; N, normal control; C, model control; S, sulfasalazine group; L, low (15 mg/kg) magnolol dose; M, medium $(30 \mathrm{mg} / \mathrm{kg})$ magnolol dose; $\mathrm{H}$, high $(60 \mathrm{mg} / \mathrm{kg})$ magnolol dose.

A

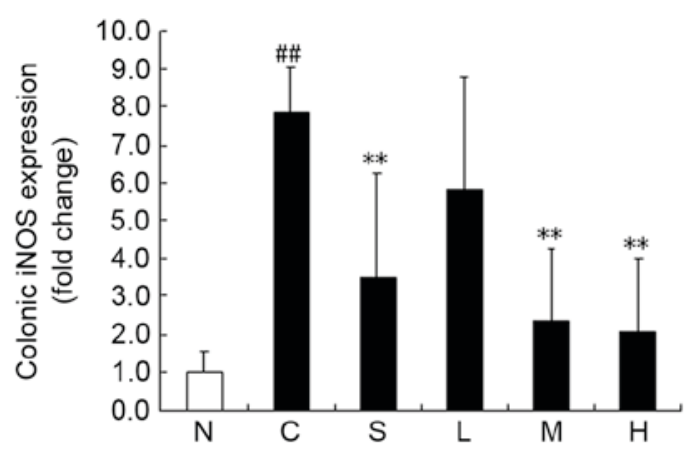

C

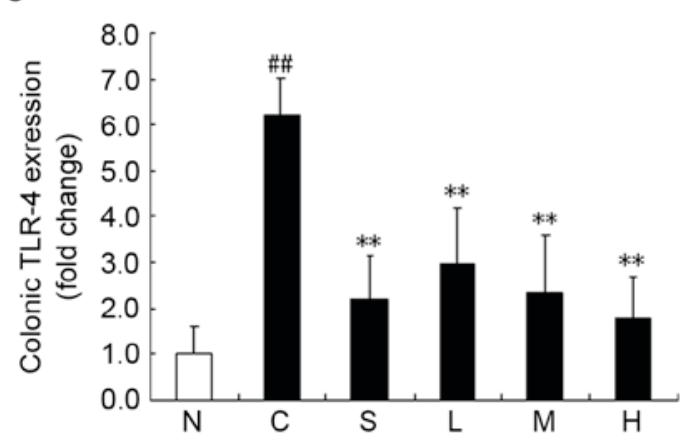

B

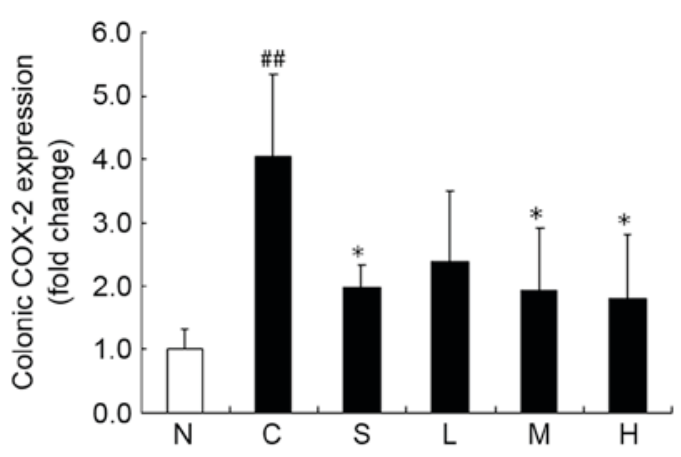

D

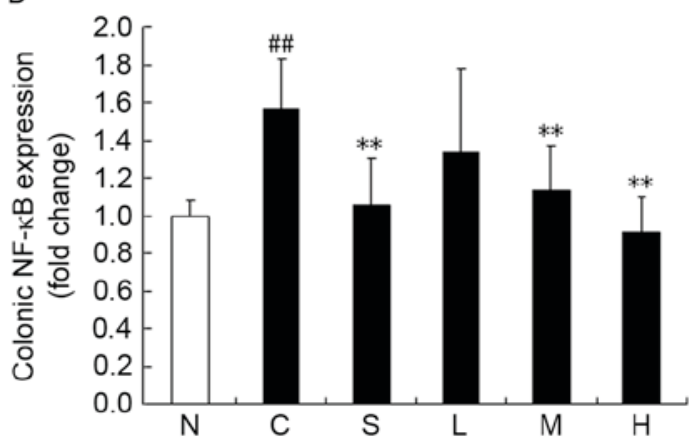

Figure 6. Magnolol ameliorates 2,4,6-trinitrobenzenesulfonic acid/ethanol-induced upregulation of inflammation-related genes. (A) iNOS, (B) COX-2, (C) TLR-4, and (D) NF-kB p65 mRNA levels. "P<0.05 and ${ }^{* *} \mathrm{P}<0.01$ vs. model control group; ${ }^{\# /} \mathrm{P}<0.01$ vs. normal control group. N, normal control; $\mathrm{C}$, model control; S, sulfasalazine group; L, low (15 mg/kg) magnolol dose; M, medium (30 mg/kg) magnolol dose; $\mathrm{H}$, high (60 mg $/ \mathrm{kg})$ magnolol dose. iNOS, inducible

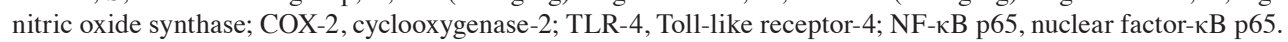

acute inflammatory process induced a reduction in thymus weight and an increase in spleen weight in TNBS-induced experimental colitis (18). Furthermore, in severe cases, the thymus almost disappeared in terms of size and cell numbers, which could be associated with an immunosuppressive state in TNBS-induced colitis (19), whereas spleen enlargement may represent a systemic inflammatory reaction that is correlated with the severity of colitis (18). In this present study, there were no significant changes regarding spleen index. However, it was demonstrated that medium and high doses of magnolol effectively improved immune function by alleviating the reduction in thymus weight, in rats with TNBS-induced colitis.
MPO is an enzyme specific to granulocyte lysosomes, which is directly correlated with the number of neutrophils (20), and is therefore used as a biochemical marker of neutrophil infiltration. Therefore, determination of MPO activity has been widely employed to detect intestinal inflammatory processes. In the present study, colonic MPO activity was significantly increased following TNBS instillation. As expected, colonic biopsies from animals that were treated with any dose of magnolol or $500 \mathrm{mg} / \mathrm{kg}$ sulfasalazine exhibited lower MPO activity than that in the TNBS control group, which indicated that magnolol treatment ameliorated neutrophil infiltration. 
A

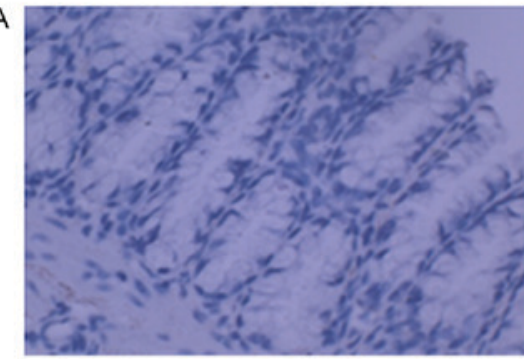

C

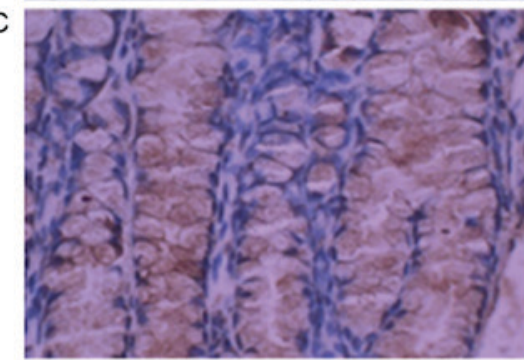

$\mathrm{E}$

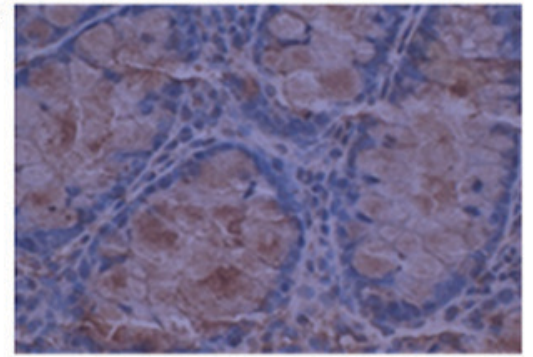

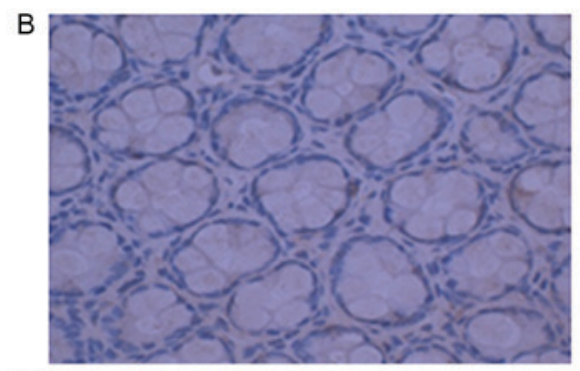

D

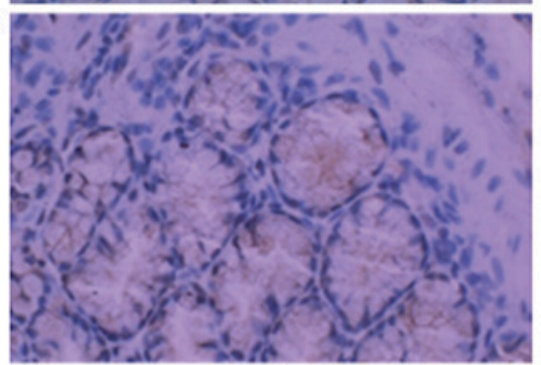

$\mathrm{F}$

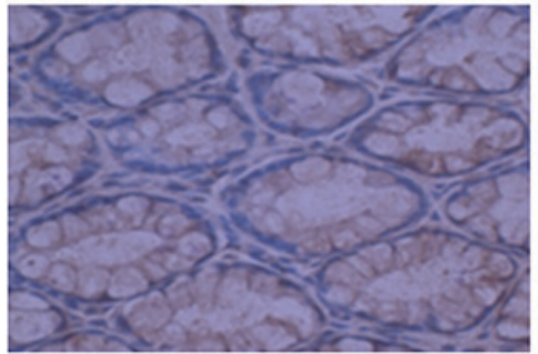

G

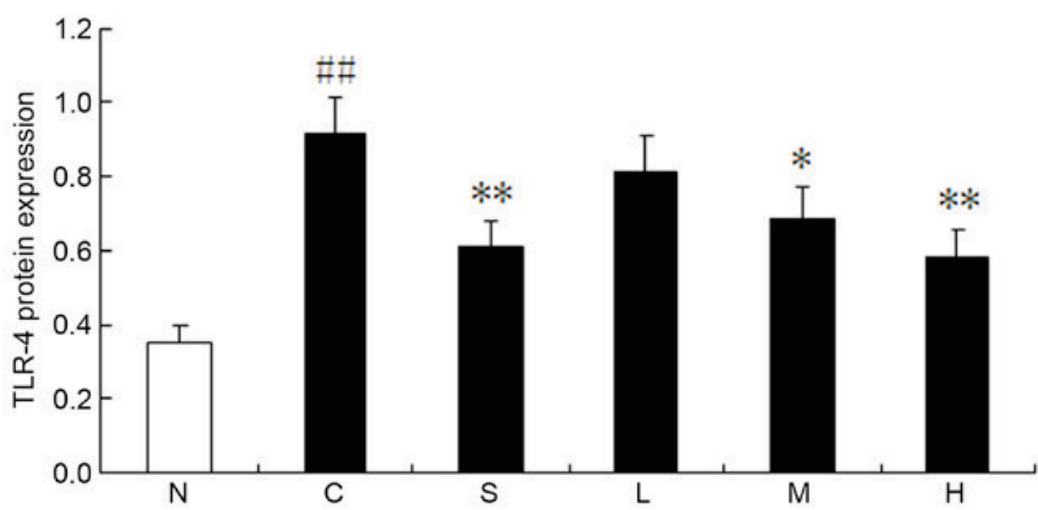

Figure 7. Magnolol ameliorates 2,4,6-trinitrobenzenesulfonic acid/ethanol-induced upregulation of TLR-4 protein expression. (A) Normal control, (B) model control and (C) sulfasalazine groups, and (D) low dose (15 mg/kg), (E) medium dose (30 mg/kg) and (F) high dose (60 mg/kg) magnolol-treated groups. (G) Pooled data from each treatment group. ${ }^{*} \mathrm{P}<0.05,{ }^{* *} \mathrm{P}<0.01$ vs. model control; ${ }^{\# \#} \mathrm{P}<0.01$ vs. normal control group. $\mathrm{N}$, normal control; $\mathrm{C}$, model control; $\mathrm{S}$, sulfasalazine group; L, low (15 mg/kg) magnolol dose; M, medium (30 mg/kg) magnolol dose; H, high (60 mg/kg) magnolol dose; TLR-4, Toll-like receptor-4.

IBD is associated with an enhanced production of the reactive metabolites of oxygen and nitrogen (21). Previous research has suggested that in human IBD, the overproduction of NO via the upregulation of iNOS may be responsible for intestinal hypomotility and subsequent bacterial overgrowth (22). Likewise, upregulation of iNOS and COX-2 expression has been demonstrated in experimental colitis (23), and was further supported in the present study by the amplification of iNOS and COX-2 mRNA expression, in colonic tissue from rats with TNBS-induced colitis. Magnolol has previously been reported to inhibit lipopolysaccharide-induced iNOS and COX-2 expression in murine macrophages, by blocking intracellular signaling pathways (24). The present study indicated that 30 and $60 \mathrm{mg} / \mathrm{kg}$ doses of magnolol significantly attenuated the elevated levels of iNOS and COX-2 gene expression, which was observed after TNBS induction.

As one of the classical indicators of the T-helper (Th)1 response, IL-6 has a fundamental role in IBD pathogenesis (25). In addition, a previous study confirmed an important role for IL- 6 in the suppression of regulatory T-cell function, and the development of pathogenic Th17 cells (26). Notably, a previous study using IL-17 receptor-A knockout mice demonstrated that IL-17 is necessary for the development of acute gut inflammation, induced by the intrarectal administration of TNBS (27). In the present study, treatment with all doses of magnolol and sulfasalazine resulted in a marked decline in serum levels of IL-6 and IL-17, demonstrating the ability of magnolol to downregulate proinflammatory cytokines. 
It has previously been reported that IBD is not induced to develop and does not progress in TLR-4 knockout animals (28). Furthermore, TLR-4 is upregulated in the intestinal mucosa of patients suffering from active IBD (29). In the present study, TLR-4 mRNA and protein levels were markedly increased upon TNBS stimulation, and were significantly reduced following treatment with the 30 and $60 \mathrm{mg} / \mathrm{kg}$ doses of magnolol.

$\mathrm{NF}-\kappa \mathrm{B}$ activation is increased in mucosal macrophages and epithelial cells in the inflamed colon of patients with IBD and animal models of IBD (30). Inhibition of NF- $\kappa$ B activation has therefore been proposed as a promising strategy for treating IBD. In the present study, TNBS provoked a marked elevation in $\mathrm{NF}-\kappa \mathrm{B}$ p 65 gene expression, and this alteration was significantly suppressed by medium and high doses of magnolol, suggesting that magnolol may effectively inhibit TLR-4-associated NF- $\kappa \mathrm{B}$ activation in IBD.

Finally, no drug-induced changes in food intake or in clinical, macroscopic, microscopic or biochemical parameters (data not shown) were observed during the 1 week course of treatment, suggesting that magnolol may be a safe product for use in rats. The strong anti-inflammatory activity of magnolol and the absence of apparent toxicity, combined with its anticancer activity (31), have made it a promising therapeutic candidate for the treatment of IBD.

In conclusion, magnolol appears to ameliorate TNBS-induced colitis in a dose-dependent manner. The beneficial effects of this compound likely involve a decrease in proinflammatory mediators (including iNOS and COX-2) and cytokines (such as IL-6 and IL-17), by inhibiting TLR-4-associated NF- $\kappa$ B activation. These findings suggest that magnolol may be a promising therapeutic agent in the clinical management of IBD.

\section{Acknowledgements}

The authors would like to gratefully acknowledge Professor Hai-Dong Li in the Department of Biochemistry and Molecular Biology at Tianjin Medical University, for providing the facilities to perform this study.

\section{References}

1. Algieri F, Zorrilla P, Rodriguez-Nogales A, Garrido-Mesa N, Bañuelos O, González-Tejero MR, Casares-Porcel M, Molero-Mesa J, Zarzuelo A, Utrilla MP, et al: Intestinal anti-inflammatory activity of hydroalcoholic extracts of Phlomis purpurea L. and Phlomis lychnitis L. in the trinitrobenzenesulphonic acid model of rat colitis. J Ethnopharmacol 146: 750-759, 2013.

2. Guo BJ, Bian ZX, Qiu HC, Wang YT and Wang Y: Biological and clinical implications of herbal medicine and natural products for the treatment of inflammatory bowel disease. Ann N Y Acad Sci 1401: 37-48, 2017.

3. Bernaskova M, Kretschmer N, Schuehly W, Huefner A, Weis R and Bauer R: Synthesis of tetrahydrohonokiol derivates and their evaluation for cytotoxic activity against CCRF-CEM leukemia, U251 glioblastoma and HCT-116 colon cancer cells. Molecules 19: 1223-1237, 2014.

4. Tsai YC, Cheng PY, Kung CW, Peng YJ, Ke TH, Wang JJ and Yen MH: Beneficial effects of magnolol in a rodent model of endotoxin shock. Eur J Pharmacol 641: 67-73, 2010.

5. Chuang DY, Chan MH, Zong Y, Sheng W, He Y, Jiang JH, Simonyi A, Gu Z, Fritsche KL, Cui J, et al: Magnolia polyphenols attenuate oxidative and inflammatory responses in neurons and microglial cells. J Neuroinflammation 10: 15, 2013.
6. Park J, Lee J, Jung E, Park Y, Kim K, Park B, Jung K, Park E, Kim J and Park D: In vitro antibacterial and anti-inflammatory effects of honokiol and magnolol against Propionibacterium sp. Eur J Pharmacol 496: 189-195, 2004.

7. Zhang M, Long Y, Sun Y, Wang Y, Li Q, Wu H, Guo Z, Li Y, Niu Y, Li C, et al: Evidence for the complementary and synergistic effects of the three-alkaloid combination regimen containing berberine, hypaconitine and skimmianine on the ulcerative colitis rats induced by trinitrobenzene-sulfonic acid. Eur J Pharmacol 651: 187-196, 2011.

8. Kang YJ, Park HJ, Chung HJ, Min HY, Park EJ, Lee MA, Shin Y and Lee SK: Wnt/ $\beta$-catenin signaling mediates the antitumor activity of magnolol in colorectal cancer cells. Mol Pharmacol 82: 168-177, 2012.

9. Witaicenis A, Luchini AC, Hiruma-Lima CA, Felisbino SL, Garrido-Mesa N, Utrilla P, Gálvez J and Di Stasi LC: Suppression of TNBS-induced colitis in rats by 4-methylesculetin, a natural coumarin: Comparison with prednisolone and sulphasalazine. Chem Biol Interact 195: 76-85, 2012.

10. Gonçalves CC, Hernandes L, Bersani-Amado CA, Franco SL, Silva JF and Natali MR: Use of propolis hydroalcoholic extract to treat colitis experimentally induced in rats by 2,4,6-trinitrobenzenesulfonic acid. Evid Based Complement Alternat Med 2013: 853976, 2013.

11. Chen YH, Lin FY, Liu PL, Huang YT, Chiu JH, Chang YC, Man KM, Hong CY, Ho YY and Lai MT: Antioxidative and hepatoprotective effects of magnolol on acetaminophen-induced liver damage in rats. Arch Pharm Res 32: 221-228, 2009.

12. Yang TC, Zhang SW, Sun LN, Wang H and Ren AM: Magnolol attenuates sepsis-induced gastrointestinal dysmotility in rats by modulating inflammatory mediators. World J Gastroenterol 14: 7353-7360, 2008.

13. Morris GP, Beck PL, Herridge MS, Depew WT, Szewczuk MR and Wallace JL: Hapten-induced model of chronic inflammation and ulceration in the rat colon. Gastroenterology 96: 795-803, 1989.

14. Tsune I, Ikejima K, Hirose M, Yoshikawa M, Enomoto N, Takei Y and Sato N: Dietary glycine prevents chemical-induced experimental colitis in the rat. Gastroenterology 125: 775-785, 2003.

15. Livak KJ and Schmittgen TD: Analysis of relative gene expression data using real-time quantitative PCR and the 2(-Delta Delta C(T)) method. Methods 25: 402-408, 2001.

16. Liu X and Wang J: Anti-inflammatory effects of iridoid glycosides fraction of Folium syringae leaves on TNBS-induced colitis in rats. J Ethnopharmacol 133: 780-787, 2011.

17. Peran L, Camuesco D, Comalada M, Bailon E, Henriksson A, Xaus J, Zarzuelo A and Galvez J: A comparative study of the preventative effects exerted by three probiotics, Bifidobacterium lactis, Lactobacillus casei and Lactobacillus acidophilus, in the TNBS model of rat colitis. J Appl Microbiol 103: 836-844, 2007.

18. Oz HS, Zhong J and de Villiers WJ: Osteopontin ablation attenuates progression of colitis in TNBS model. Dig Dis Sci 57: 1554-1561, 2012.

19. Fritsch Fredin M, Elgbratt K, Svensson D, Jansson L, Melgar S and Hultgren Hörnquist E: Dextran sulfate sodium-induced colitis generates a transient thymic involution-impact on thymocyte subsets. Scand J Immunol 65: 421-429, 2007.

20. Xing JF, Sun JN, Sun JY, You CY, Dong K, Lv J and Dong YL: Protective effects of 3,4-oxo-isopropylidene-shikimic acid on experimental colitis induced by trinitrobenzenesulfonic acid in rats. Dig Dis Sci 57: 2045-2054, 2012.

21. Conner EM, Brand SJ, Davis JM, Kang DY and Grisham MB: Role of reactive metabolites of oxygen and nitrogen in inflammatory bowel disease: Toxins, mediators, and modulators of gene expression. Inflamm Bowel Dis 2: 133-147, 1996.

22. Szalai Z, Szász A, Nagy I, Puskás LG, Kupai K, Király A, Berkó AM, Pósa A, Strifler G, Baráth Z, et al: Anti-inflammatory effect of recreational exercise in TNBS-induced colitis in rats: Role of NOS/HO/MPO system. Oxid Med Cell Longev 2014: 925981, 2014.

23. Sun P, Zhou K, Wang S, Li P, Chen S, Lin G, Zhao Y and Wang T: Involvement of MAPK/NF- $\mathrm{BB}$ signaling in the activation of the cholinergic anti-inflammatory pathway in experimental colitis by chronic vagus nerve stimulation. PLoS One 8: e69424, 2013.

24. Lai CS, Lee JH, Ho CT, Liu CB, Wang JM, Wang YJ and Pan MH: Rosmanol potently inhibits lipopolysaccharide-induced iNOS and COX-2 expression through downregulating MAPK, NF-kappaB, STAT3 and C/EBP signaling pathways. J Agric Food Chem 57: 10990-10998, 2009. 
25. Du L, Tang H, Ma Z, Xu J, Gao W, Chen J, Gan W, Zhang Z, Yu X, Zhou X and Hu X: The protective effect of the recombinant 53-kDa protein of Trichinella spiralis on experimental colitis in mice. Dig Dis Sci 56: 2810-2817, 2011.

26. Xiong J, Lin YH, Bi LH, Wang JD, Bai Y and Liu SD: Effects of interleukin- 4 or interleukin-10 gene therapy on trinitrobenzenesulfonic acid-induced murine colitis. BMC gastroenterol 13: $165,2013$.

27. Caprioli F, Pallone F and Monteleone G: Th17 immune response in IBD: A new pathogenic mechanism. J Crohns Colitis 2: 291-295, 2008.

28. Joh EH, Lee IA, Han SJ, Chae S and Kim DH: Lancemaside A ameliorates colitis by inhibiting NF-kappaB activation in TNBS-induced colitis mice. Int J Colorectal Dis 25: 545-551, 2010.
29. Himmel ME, Hardenberg G, Piccirillo CA, Steiner TS and Levings MK: The role of T-regulatory cells and Toll-like receptors in the pathogenesis of human inflammatory bowel disease. Immunology 125: 145-153, 2008.

30. Pasparakis M: IKK/NF-kappaB signaling in intestinal epithelial cells controls immune homeostasis in the gut. Mucosal Immunol 1 (Suppl 1): S54-S57, 2008.

31. Seo JU, Kim MH, Kim HM and Jeong HJ: Anticancer potential of magnolol for lung cancer treatment. Arch Pharm Res 34: 625-633, 2011.

This work is licensed under a Creative Commons Attribution-NonCommercial-NoDerivatives 4.0 International (CC BY-NC-ND 4.0) License. 